\title{
Anterior Cruciate Ligament Reconstruction: A Multicenter Prospective Cohort Study Evaluating 3 Different Grafts Using Same Bone Drilling Method
}

Joan Leal-Blanquet, $\mathrm{MD}, \mathrm{PhD},{ }^{*}$ Eduard Alentorn-Geli, $\mathrm{MD}, \mathrm{MS}, \mathrm{PhD},{ }^{*}$ Josep Tuneu, MD, PhD, $\dagger$ Joan Ramon Valentí, MD, PhD, $\ddagger$ and Antonio Maestro, MD, PhD§

*Department of Orthopedic Surgery, Hospital de l'Esperança - Parc de Salut MAR, Barcelona, Spain

†Department of Orthopedic Surgery, Centro Medico Teknon, Barcelona, Spain. $\ddagger$ Department of Orthopedic Surgery, Clínica Universitaria de Navarra, Pamplona, Spain.

$\S$ Department of Orthopedic Surgery, Clínica FREMAP, Gijón, Spain.

OBJECTIVE: To compare the clinical outcomes after anterior cruciate ligament (ACL) reconstruction with bone-patellar tendon-bone autograft (BPTBAu), BPTB allograft (BPTBAll), or hamstring (semitendinosus-gracilis) tendon autograft (HTAu), performing bone drilling with same methods in terms of transtibial drilling, orientation, positioning, and width of femoral and tibial tunnels.

DESIGN: Multicenter prospective cohort study (level of evidence II).

SETTING: Departments of Orthopedic Surgery of Centro Médico Teknon (Barcelona, Spain) Clínica Universitaria de Navarra (Navarra, Spain), and Clínica FREMAP (Gijón, Spain).

PATIENTS: All patients with ACL tears attending 3 different institutions between January 2004 and June 2006 were approached for eligibility and those meeting inclusion criteria finally participated in this study.

INTERVENTION: Each institution was assigned to perform a specific surgical technique. Patients were prospectively followed after undergoing ACL reconstruction with BPTBAu, BPTBAll, or HTAu, with a minimum follow-up of 24 months.

MAIN OUTCOME MEASURES: Included knee laxity and International Knee Documentation Committee (IKDC) score. Knee laxity was assessed with the KT-1000 arthrometer (evaluated with neutral and external rotation positions) and both Lachman and pivot shift tests. Additional outcomes included main symptoms (anterior knee pain, swelling, crepitation, and instability), disturbance in knee sensation, visual analogue scale (VAS) for satisfaction with surgery, range of motion (ROM), and isokinetic knee strength.

RESULTS: There were no significant differences among the 3 groups for any of the clinical outcomes, except for a slightly greater KT-1000-measured knee laxity in external rotation in the BPTBAu compared with the other groups. All patients demonstrated grade A or B of the IKDC. The mean VAS for satisfaction with surgery in all patients was 8.5.

CONCLUSIONS: The selection of the surgical technique for ACL reconstruction may be based on the surgeon's preferences.

KEY WORDS: anterior cruciate ligament reconstruction, bone-patellar tendon-bone, hamstrings autograft, multicenter study, bone drilling methods

Corresponding Author: Joan Leal-Blanquet, MD, PhD, Department de Cirugía Ortopédica i Traumatología, Hospital de l’Esperança, Ptge. de Sant Josep de la Muntanya 12, Barcelona 08024, Spain (jleal@parcdesalutmar.cat). 


\section{INTRODUCTION}

Anterior cruciate ligament (ACL) tears are one of the most common severe injuries in sports worldwide. Anterior cruciate ligament reconstructions can be performed using different techniques depending on the type of graft (autograft or allograft), the donor site [mainly the patellar tendon or the hamstring tendon (HT)], the morphology of the new ligament (single bundle or double bundle), the fixation of the graft, and the bone drilling method. The bone-patellar tendon-bone autograft (BPTBAu) is still the preferred method, although the use of HT autograft (HTAu) is rising in popularity. ${ }^{1}$ The BPTBAu has bone plugs on each end of the graft that may provide an excellent fixation due to its rapid incorporation within bone tunnels. ${ }^{2}$ Bone-to-bone attachment was suggested to offer greater knee stability compared with HT grafts, ${ }^{3,4}$ but no significant differences on knee laxity between both types of graft have been reported in the literature. ${ }^{5-15}$ Less controversy exists with the fact that the BPTB ACL reconstruction can be associated with increased donor-site morbidity. ${ }^{3,8,10,13,16,17}$ Overall, the answer regarding which technique provides the best clinical outcomes in ACL reconstruction has not been determined. ${ }^{15,18-}$ 23

Previous studies in ACL reconstruction with comparisons among the BPTBAu, BPTB allograft (BPTBAll), and HTAu have been conducted by several authors, but many of them neither mentioned the bone drilling method nor compared these 3 techniques in the same study. ${ }^{15,18,24-32}$ Variations in bone drilling methods in terms of portal use, orientation (angulation of the tunnel), positioning (location of the entry point of the tunnel), and width of femoral and tibial tunnels (or variations in graft fixation as well) may introduce confounding factors when comparing the clinical outcomes among different surgical techniques. ${ }^{33-36}$ Taylor et $\mathrm{al}^{34}$ recently published a comparative study between the use of BPTB and HTAu for ACL reconstruction using similar graft fixation techniques, but the position of the femoral tunnel in the sagittal plane was not exactly the same among the groups. Although femoral tunnels in these groups were drilled transtibially with similar coronal plane orientation, differences in the position of the tunnel in the sagittal plane may create a potential source of bias.

The purpose of this study was to compare the clinical outcomes after ACL reconstruction with the most commonly used grafts (BPTBAu, BPTBAll, or HTAu) while performing bone drilling with the same methods in terms of transtibial drilling, orientation, positioning, and width of femoral and tibial tunnels. It was hypothesized that the 3 groups would demonstrate no significant differences in the clinical outcomes.

\section{MATERIALS AND METHODS}

Between January 2004 and June 2006, all patients with a history and physical examination consistent with an ACL tear and confirmed with magnetic resonance imaging were approached for eligibility at 3 different institutions. All patients fulfilled the following inclusion criteria: (1) aged between adolescents with closed physes and 45 years old, (2) isolated ACL tear, (3) no associated meniscal tears or chondral lesions, and (4) no past injuries to the contralateral ACL. We applied restrictive inclusion criteria to minimize potential confounding factors and to compare a highly homogeneous sample. All patients gave consent to participate in this study, which was accepted by the institutional review board clearance in all the 3 centers before commencement. 
A multicenter prospective cohort study (level II evidence) with a minimum follow-up of 24 months was conducted. Twenty-four months was considered enough time for both a safe return to sports and a "ligamentization"process to occur. ${ }^{37,38}$ Inclusion of patients was performed during the data collection period. However, in 1 center, this period was not enough to reach the minimum sample size to detect significant differences. In this case, the collection period was extended until a sufficient sample size was reached. Each institution was assigned to perform 1 of the 3 surgical techniques (BPTBAu, BPTB All, or HTAu) based on the presence of the surgeon with most experience in that technique.

The drilling of the femoral tunnel was performed through the transtibial technique with the same orientation ( $30^{\circ}$ in the coronal plane from the vertical line), positioning (11o'clock or 1-o'clock position), and width $(8 \mathrm{~mm})$ in all the groups (Figure). This tunnel was performed with an offset femoral guide and positioned in the central point of the femoral ACL footprint. The tibial tunnel was positioned in the middle of the tibial ACL footprint, and it was done with an angle of $55^{\circ}$ in the sagittal plane in all the patients. The fixation of the graft into both the femur and the tibia was performed with the use of bioabsorbable interference screws in BPTB groups. In the HT group, the femoral fixation was performed using the Rigid-Fix (DePuy Mitek, Raynham, Massachusetts) pins technique, whereas bioabsorbable interference screws were used for tibial fixation. An independent physician performed all postoperative assessments in all the 3 institutions.

Postoperative assessments of clinical and functional variables were obtained for each included patient. The main outcome of this study was the side-to-side differences in knee stability (healthy knee - operated knee) measured with the KT-1000 arthrometer (MEDmetric, San Diego, California), taken at manual maximum, and evaluated with neutral and external rotation positions, each 1 taken twice, 30 minutes apart. The patients laid down during the 30 minutes between both measures. All knees were conditioned with multiple anterior/posterior draw maneuvers before the final measure was taken. The value of knee stability was obtained by reading off the dial by the assessor. Other measures of side-to-side knee stability were also obtained through the subjective Lachman and pivot shift tests. The outcomes of the Lachman test were given as no, mild (1-5 mm), moderate $(6-10 \mathrm{~mm})$, or severe $(>10 \mathrm{~mm})$ side-to-side differences. The outcomes of the pivot shift test were also given as no, mild (glide), moderate (clunk), or severe (gross) side-to-side differences. The overall (subjective and objective components) International Knee Documentation Committee (IKDC) score was also assessed. Additionally, data on major symptoms [anterior knee pain, swelling, crepitation, and instability, reported as the presence (yes) or absence (no) of each 1], disturbance in knee sensation evaluated through palpation with a needle [reported as the presence (yes) or absence (no) of sensation], visual analogue scale (VAS) for satisfaction with surgery in a 0 to 10 scale ( 0 meaning worst and 10 meaning best results), and lack of active range of motion (ROM) were collected from the anamnesis and physical examination. The ROM was measured with a goniometer, and differences in $>5^{\circ}$ in either extension or flexion with respect to the contralateral side were considered abnormal [reported as the presence (yes) or absence (no) of complete ROM]. Flexion and extension were assessed separately in the supine position. The isokinetic knee strength was measured using the Biodex System 3 Isokinetic Dynamometer (Biodex Medical Systems, Shirley, New York). The patient was placed in the upright sitting-back position at approximately $80 \%$ with fixed thorax, pelvis, and contralateral femur. The training protocol consisted of 5 repetitions at $60^{\circ}$ per second and 10 repetitions at 1800 per second for the knee extensors concentric/knee flexors concentric mode, and 5 repetitions for both angular velocities for the knee extensors eccentric/knee flexors eccentric mode. The warm-up included 5 
minutes of static bike and 10 submaximal repetitions in concentric/eccentric mode (angular velocity 600/s and 1800/s). The outcome measured for isokinetic knee strength was the peak torque (in newton meter), but the values were reported as the percentage of deficit in peak torque between the operated and healthy leg for both quadriceps and hamstrings [percentage of deficit in peak torque $=$ (peak torque operated/peak torque healthy) x 100].

The rehabilitation protocol was standardized for all the 3 involved institutions. All patients began ROM exercises during the first postoperative week. The ROM progressively increased in the subsequent week until a complete ROM was reached by weeks 2 to 3 . Strength training began with isometric exercises during the first postoperative week, progressively increasing the intensity until isokinetic exercises were incorporated by weeks 3 to 4 . The patients began walking with 2 crutches in the first postoperative week, but 1 crutch was removed by week 2 , and unassisted walk was allowed by week 4 . Proprioceptive training through closed kinetic chains exercises began in the second postoperative week. Cycling and swimming activities were introduced by week 6, whereas jogging was not allowed until the third month. Sports were progressively introduced by the fifth postoperative month.

\section{STATISTICAL ANALYSIS}

Descriptive statistics were used to summarize the demographic characteristics of the 3 groups. A 1-way analysis of variance was used to compare quantitative demographic data among the groups. The $\chi^{2}$ statistical test was used for categorical variables, whereas Kruskal-Wallis test was used for quantitative variables. An intraobserver reproducibility analysis was conducted for the KT-1000 arthrometer measurement using the intraclass correlation coefficient (ICC). An "a priori” sample size calculation was performed in this study. This analysis was based on the main outcome knee stability KT-1000. The minimum difference being clinically relevant for anterior-posterior knee laxity (KT-1000 assessment) was established in $3 \mathrm{~mm}$ for either neutral or external rotation. Based on the previous literature, the mean of side-to-side differences in KT-1000 for BPTBAu, BPTBAll, and HTAu were $1.96 \mathrm{~mm}, 4.5 \mathrm{~mm}$, and 2.5, with a sigma of $2 .{ }^{19-22,32}$ This elicited a minimum sample size of 15 per group to detect significant differences among the groups at an alpha level of 0.05 (power of $80 \%$ ). All statistical tests were performed using the SPSS version 15 (SPSS, Inc, Chicago, Illinois).

\section{RESULTS}

A total of 51 patients met the inclusion criteria (15 in the BPTBAu group, 16 in the BPTBAll group, and 20 in the HTAu group). The follow-up [mean (SD)] was 36 (8), 34 (5), 32 (6) months for the BPTBAu, BPTBAll, and HTAu groups, respectively. No patients declined participation in the study, and none were lost to follow-up. No significant differences in the demographic characteristics were found among the groups (Table 1$)$, including similar male to female ratios $(\mathrm{P}=0.906)$. No statistically significant differences were found for the KT-1000, Lachman, and pivot shift tests among the groups, except for a slightly greater knee laxity measured through the KT1000 in external rotation in the BPTBAu (mean side-to-side difference of $-1.13 \mathrm{~mm}$ ) compared with the BPTBAll (mean side-to-side difference of $0.38 \mathrm{~mm}$ ) and HTAu groups (mean side-toside difference of $0.05 \mathrm{~mm}$ ) in the first of the 2 evaluations (Table 2). No patients 
demonstrated moderate or severe side-to-side differences in the Lachman or pivot shift tests, neither graded C or D in the IKDC score. Sixty-five percent of values of the KT1000 knee laxity were the same when comparing both measures taken 30 minutes apart (mean differences never above $1.2 \mathrm{~mm}$ ). The intraclass correlation between each measure and its corresponding assessment after 30 minutes was 0.92 (95\% confidence interval (CI) 0.87-0.96) for right knee in neutral position, 0.95 (0.92-0.97) for right knee in external rotation, 0.94 (0.9-0.97) for left knee in neutral position, and $0.96(0.92-0.97)$ for left knee in external rotation. No significant differences were found for the IKDC score among the 3 groups. All patients demonstrated grade A or B of the IKDC assessment. No other statistically significant differences were found for major symptoms (anterior knee pain, swelling, crepitation, and instability), disturbance in knee sensation, lack of active ROM, the VAS for satisfaction with surgery, and isokinetic knee strength among the groups (Tables 3 and 4). No disturbances in knee sensation were found in the allograft group, whereas we obtained disturbances in knee sensation in 3 and 2 patients in the BPTBAu and HTAu groups, respectively $(\mathrm{P}=0.2)$. Fourteen patients $(93.3 \%)$ in the BPTBAu group, 16 patients (100\%) in the BPTBAll group, and 19 patients (95\%) in the HTAu group demonstrated a complete ROM $(\mathrm{P}=0.61)$. The mean VAS for satisfaction in all patients was 8.5.

\section{DISCUSSION}

The most important finding of this study was the absence of significant differences in any of the outcomes after ACL reconstruction with the most commonly used grafts (BPTBAu, BPTBAll, or HTAu) while performing bone drilling with the same methods in terms of transtibial drilling, and orientation, positioning and width of femoral and tibial tunnels. There are numerous studies comparing a wide variety of ACL reconstruction techniques, ${ }^{15,18-23,32}$ without a clear consensus on which one provides the best clinical outcomes. Unfortunately, many studies have not controlled for the influence of potential confounding factors, such as variations in the graft fixation or bone drilling methods. In addition, the level of evidence of studies dealing with ACL reconstruction and the control of potential sources of bias is not the best. ${ }^{15}$ In the current study, we compared clinical outcomes after ACL reconstruction by use of the most commonly employed grafts (BPTBAu, BPTBAll, or HTAu) with the same operative methods. We conducted a multicenter prospective cohort study where no statistically significant differences in terms of knee stability, overall IKDC, knee symptoms, disturbances in knee sensation, satisfaction with surgery, ROM, and isokinetic knee strength were found among the groups.

The demographic characteristics of our sample are comparable with those from other studies. ${ }^{18-23,32}$ In addition, the assessed clinical outcomes have been used in the majority of articles published in the ACL reconstruction literature. Although other authors have reported longer followup, ${ }^{5,11,13,14,16,24}$ a follow-up of 2 years may be considered long enough to report clinical outcomes in ACL reconstruction. ${ }^{37,38}$ In fact, most studies report the short-term and midterm instead of the long-term follow-up. ${ }^{15,32}$

The results of this study are consistent with the existing literature when analyzing both the BPTBAu-HTAu and the BPTBAll-BPTBAu comparisons. Other studies comparing the BPTBAu versus HTAu did not demonstrate statistically significant differences in the overall IKDC score, ${ }^{5,7-9,14,17}$ knee stability measures, ${ }^{5-11,14}$ ROM ${ }^{14,17}$ and muscle strength and/or single-leg hop test. ${ }^{5-9,14,17}$ Similarly, studies comparing the BPTBAu and BPTBAll 
did not find significant differences in the overall IKDC, ${ }^{24,26}$ knee stability measures, ${ }^{26,27,29-31,39}$ ROM, ${ }^{24,26,27,29-31,39}$ and muscle strength and/or single-leg hop test. $^{24,26,30,31,39}$ The same conclusions can be drawn when considering the results of those studies using the same bone drilling method. ${ }^{5-7,16,24}$

Controversial data do exist for some of these clinical outcomes. Feller and Webster ${ }^{3}$ and Anderson et $\mathrm{al}^{4}$ found significant differences in knee stability between the use of the BPTBAu and the HTAu techniques. However, caution must be taken when comparing these results with those obtained in the present study because the 2 studies were conducted using different bone drilling methods between the groups. ${ }^{3,4}$ The absence of differences for disturbances in knee sensation and, in general, for donor-site morbidity between our study groups is only partially supported by the literature. ${ }^{4,5,14}$ The majority of studies have found higher donor-site morbidity in the BPTBAu group compared with the HTAu group. ${ }^{3,8,10,13,16,17}$ However, it was found that these differences may disappear with time. ${ }^{15}$ Patients with BPTBAu complained of disturbances in knee sensation more than in the other 2 groups, although the difference was not found to be statistically significant.

In a recent comprehensive systematic review of randomized controlled trials in ACL reconstruction, Samuelsson et $\mathrm{al}^{15}$ found no differences between the use of the BPTBAu or HTAu in terms of knee laxity, clinical outcomes, time to return to sports, patellofemoral crepitation, performance on the 1-leg hop test, ROM, thigh circumference, and anterior knee sensory deficits. The authors reported that the BPTBAu was found to possibly cause more knee osteoarthritis compared with the HT graft, but the latter had more tunnel widening compared with the former. Tunnel widening was not associated with poor clinical outcomes or laxity. ${ }^{15}$ Although they reported an excellent and guiding review, data on bone drilling methods were not specifically considered in the data extraction sheet. Hence, the influence of this potential confounding factor in the reviewed studies remains unknown.

We are unable to explain why patients in the BPTBAu group demonstrated a greater KT1000-measured knee laxity in external rotation during the first assessment because the tibial tunnel position was equal in each group. Nonetheless, the mean values in external rotation and those values during the second assessment were not statistically different. In addition, we believe that the existing difference in KT-1000 values in external rotation among the groups during the first assessment was not clinically relevant because group means were only differing in $1 \mathrm{~mm}$. Also, the values obtained for the isokinetic knee strength are difficult to interpret. However, values of less than $15 \%$ for percentage of deficit of peak torque between both the operated and healthy legs are considered to be irrelevant. ${ }^{40}$ Moreover, these are normal percentages of deficit for patients 1 year after the surgery of ACL reconstruction. ${ }^{40}$

We believe that this study has several strengths. First, the design was conceived to control for potential confounding factors in the outcomes of ACL reconstruction. Essentially, this study attempted to systematize the bone drilling method in terms of same portal use, orientation (angulation of the tunnel), positioning (location of the entry point of the tunnel), and width of femoral and tibial tunnels among the groups. Modifications in bone drilling methods may affect knee laxity values. Second, the use of a homogeneous sample trying to isolate ACL tears from concomitant or past injuries in either the ipsilateral or contralateral knees also allowed the control of potential sources of biases. Beyond concerns of a potential decrease in the external validity, strict inclusion criteria 
may increase the internal validity and, therefore, the validity of the conclusions made from this sample. Third, the high intraobserver reproducibility for one of the most important outcomes (the objectively measured knee laxity) represents the lack of systematic errors in this relevant parameter.

On the other hand, we recognize 3 potential limitations to this study. First and foremost, the patients were not randomly assigned to the surgical treatment. However, we believe that the strict inclusion criteria and the absence of differences in the demographic characteristics among the groups may have restricted this limitation. In addition, by ensuring the most expertise for ACL reconstruction with each technique, the influence of experience-related variability was diminished. Second, although the sample size seems small, based on the sample size calculation, it was sufficient to detect significant differences with an alpha level of 0.05 and a power of $80 \%$. Third, the fixation of the graft with the Rigid-Fix in the HT group may not be equivalent to interference screws used in the other 2 groups. However, the impact of this difference in our study may be limited for 2 main reasons. First, some authors have found no differences between the fixation of HT with the use of bioabsorbable pins or the interference screw. ${ }^{41}$ Second, other parameters of ACL reconstruction influencing the clinical outcomes (morphology of the ligament and drilling methods) were defined and systematized to ensure equal conditions among the 3 groups. The reason why the Rigid-Fix system was used in this study is based on the previous literature, where it was found that interference screws provided an inferior fixation for the HT graft compared with the BPTB graft. ${ }^{42}$ The fact that controversial data do exist with this topic makes us consider that the different fixation methods is not a major concern.

\section{CONCLUSIONS}

We found no significant differences in any of the outcomes among the 3 groups. The use of all the 3 studied techniques elicited excellent clinical outcomes. The results of this study are consistent with those existing in the literature. The selection of the surgical technique for ACL reconstruction may be based on the surgeon's preferences and expertise.

\section{REFERENCES}

1. Duquin TR, Wind WM, Fineberg MS, et al. Current trends in anterior cruciate ligament reconstruction. J Knee Surg. 2009;22:7-12.

2. Sherman $\mathrm{OH}$, Banffy MB. Anterior cruciate ligament reconstruction: which graft is best? Arthroscopy. 2004;20:974-980.

3. Feller JA, Webster KE. A randomized comparison of patellar tendon and hamstring tendon anterior cruciate ligament reconstruction. Am J Sports Med. 2003;31:564-573.

4. Anderson AF, Snyder RB, Lipscomb AB. Anterior cruciate ligament reconstruction. A prospective randomized study of three surgical methods. Am J Sports Med. 2001;29:272-279.

5. Sajovic M, Vengust V, Komadina R, et al. A prospective, randomized comparison of semitendinosus and gracilis tendon versus patellar tendon autografts for anterior cruciate ligament reconstruction. Five-year follow-up. Am J Sports Med. 2006;34:1933-1940. 
6. Shaieb MD, Kan DM, Chang SK, et al. A prospective randomized comparison of patellar tendon versus semitendinosus and gracilis tendon autografts for anterior cruciate ligament reconstruction. Am J Sports Med. 2002;30:214-220.

7. Beard DJ, Anderson JL, Davies S, et al. Hamstrings versus patella tendon for anterior cruciate ligament reconstruction: a randomised controlled trial. Knee. 2001;8:45-50.

8. Aglietti P, Giron F, Buzzi R, et al. Anterior cruciate ligament reconstruction: bone-patellar tendon-bone compared with double semitendinosus and gracilis tendon grafts. A prospective, randomized clinical trial. JBone Joint Surg Am. 2004;86:2143-2155.

9. Ejerhed L, Kartus J, Sernert N, et al. Patellar tendon or semitendinosus tendon autografts for anterior cruciate ligament reconstruction? A prospective randomized study with a two-year follow-up. Am J Sports Med. 2003;31:19-25.

10. Aune AK, Holm I, Risberg MA, et al. Four-strand hamstring tendon autograft compared with patellar tendon-bone autograft for anterior cruciate ligament reconstruction. A randomized study with two-year follow-up. Am JSports Med. 2001;29:722-728.

11. Ibrahim SAR, Al-Kussary IM, Al-Misfer ARK, et al. Clinical evaluation of arthroscopically assisted anterior cruciate ligament reconstruction: patellar tendon versus gracilis and semitendinosus autograft. Arthroscopy. 2005;21:412-417.

12. Laxdal G, Kartus J, Hansson L, et al. A prospective randomized comparison ofbone-patellar tendon-bone and hamstring grafts for anterior cruciate ligament reconstruction. Arthroscopy. 2005;21:34-42.

13. Matsumoto A, Yoshiya S, Muratsu $\mathrm{H}$, et al. A comparison of bone-patellar tendon-bone and bone-hamstring tendon-bone autografts for anterior cruciate ligament reconstruction. Am J Sports Med. 2006;34:213-219.

14. Lidén M, Ejerhed L, Sernert N, et al. Patellar tendon or semitendinosus tendon autografts for anterior cruciate ligament reconstruction. A prospective, randomized study with a 7-year follow-up. Am J Sports Med. 2007;35:740-748.

15. Samuelsson K, Andersson D, Karlsson J. Treatment of anterior cruciate ligament injuries with special reference to graft type and surgical technique: an assessment of randomized controlled trials. Arthroscopy. 2009;25:1139-1174.

16. Pinczewski LA, Lyman J, Salmon LJ, et al. A 10-year comparison of anterior cruciate ligament reconstructions with hamstring tendon and patellar tendon autograft: a controlled, prospective trial. Am J Sports Med. 2007;35:564-574.

17. Maletis GB, Cameron SL, Tengan JJ, et al. A prospective randomized study of anterior cruciate ligament reconstruction. A comparison of patellar tendon and quadrupled-strand semitendinosus/gracilis tendons fixed with bioabsorbable interference screws. Am J Sports Med. 2007;35: 384-394.

18. Krych AJ, Jackson JD, Hoskin TL, et al. A meta-analysis of patellar tendon autograft versus patellar tendon allograft in anterior cruciate ligament reconstruction. Arthroscopy. 2008;24:292-298.

19. Freedman KB, D’Amato MJ, Nedeff DD, et al. Arthroscopic anterior cruciate ligament reconstruction: a metaanalysis comparing patellar tendon and hamstring tendon autografts. Am JSports Med. 2003;31:2-11.

20. Yunes M, Richmond JC, Engels EA, et al. Patellar versus hamstring tendons in anterior cruciate ligament reconstruction: a meta-analysis. Arthroscopy. 2001;17:248-257.

21. Goldblatt JP, Fitzsimmons SE, Balk E, et al. Reconstruction of the anterior cruciate ligament: meta-analysis of patellar tendon versus hamstring tendon autograft. Arthroscopy. 2005;21:791-803. 
22. Spindler KP, Kuhn JE, Freedman KB, et al. Anterior cruciate ligament reconstruction autograft choice: bone-tendon-bone versus hamstring. Does it really matter? A systematic review. Am J Sports Med. 2004;32: 1986-1995.

23. Herrington L, Wrapson C, Matthews $M$, et al. Anterior cruciate ligament reconstruction, hamstring versus bone-patella tendon-bone grafts: a systematic literature review of outcome from surgery. Knee. 2005;12:41-50.

24. Kleipool AEB, Zijl JAC, Willems WJ. Arthroscopic anterior cruciate ligament reconstruction with bone-patellar tendon-bone allograft or autograft. A prospective study with an average follow up of 4 years. Knee Surg Sports Traumatol Arthrosc. 1998;6:224-230.

25. Poehling GG, Curl WW, Lee CA, et al. Analysis of outcomes of anterior cruciate ligament repair with 5-year follow-up: allograft versus autograft. Arthroscopy. 2005;21:774-785.

26. Barrett G, Stokes D, White M. Anterior cruciate ligament reconstruction in patients older than 40 years. Allograft versus autograft patellar tendon. Am J Sports Med. 2005;33:1505-1512.

27. Chang SKY, Egami DK, Shaieb MD, et al. Anterior cruciate ligament reconstruction: allograft versus autograft. Arthroscopy. 2003;19:453-462.

28. Harner CD, Olson E, Irrgang JJ, et al. Allograft versus autograft anterior cruciate ligament reconstruction. Three- to 5-year outcome. Clin Orthop Relat Res. 1996;324:134-144.

29. Peterson RK, Shelton WR, Bomboy AL. Allograft versus autograft patellar tendon anterior cruciate ligament reconstruction: a 5-year followup. Arthroscopy. 2001;17:9-13.

30. Shelton WR, Papendick L, Dukes AD. Autograft versus allograft anterior cruciate ligament reconstruction. Arthroscopy. 1997;13:446-449.

31. Stringham DR, Pelmas CJ, Burks RT, et al. Comparison of anterior cruciate ligament reconstructions using patellar tendon autograft or allograft. Arthroscopy. 1996;12:414-421.

32. Alentorn-Geli E, Lajara F, Samitier G, et al. The transtibial versus the anteromedial portal technique in the arthroscopic bone-patellar tendonbone anterior cruciate ligament reconstruction. Knee Surg Sports Traumatol Arthrosc. 2010;18:1013-1037.

33. Scopp JM, Jasper LE, Belkoff SM, et al. The effect of oblique femoral tunnel placement on rotational constraint of the knee reconstructed using patellar tendon autografts. Arthroscopy. 2004;20:294-299.

34. Taylor DC, DeBerardino TM, Nelson BJ, et al. Patellar tendon versus hamstring tendon autografts for anterior cruciate ligament reconstruction. A randomized controlled trial using similar femoral and tibial fixation methods. Am J Sports Med. 2009;37:1946-1957.

35. Loh JC, Fukuda Y, Tsuda E, et al. Knee stability and graft function following anterior cruciate ligament reconstruction: comparison between 11 o'clock and 10 o'clock femoral tunnel placement. Arthroscopy. 2003; 19:297-304.

36. Hantes ME, Zachos VC, Liantsis A, et al. Differences in graft orientation using the transtibial and anteromedial portal technique in anterior cruciate ligament reconstruction: a magnetic resonance imaging study. Knee Surg Sports Traumatol Arthrosc. 2009;17:880-886.

37. Cascio BM, Culp L, Cosgarea AJ. Return to play after anterior cruciate ligament reconstruction. Clin Sports Med. 2004;23: 395-408. 
38. Falconiero RP, DiStefano VJ, Cook TM. Revascularization and ligamentization of autogenous anterior cruciate ligament grafts in humans. Arthroscopy. 1998;14:197-205.

39. Victor J, Bellemans J, Witvrouw E, et al. Graft selection in anterior cruciate ligament reconstruction. A prospective analysis of patellar tendon autografts compared with allografts. Int Orthop. 1997;21:93-97.

40. Dvir Z, ed. Isokinetics. Muscle Testing, Interpretation and Clinical Applications. 2nd ed. Edinburgh, United Kingdom: Churchill-Livingstone; 2004.

41. Stengel D, Casper D, Bauwens $K$, et al. Bioresorbable pins and interference screws for fixation of hamstring tendon grafts in anterior cruciate ligament reconstruction surgery: a randomized controlled trial. Am J Sports Med. 2009;37:1692-1698.

42. Aune AK, Ekeland A, Cawley PW. Interference screw fixation of hamstring versus patellar tendon for anterior cruciate ligament re-construction. Knee Surg Sports Traumatol Arthrosc. 1998;6:99-102.

The authors state that no funding was received for this study. The authors report no conflicts of interest. 


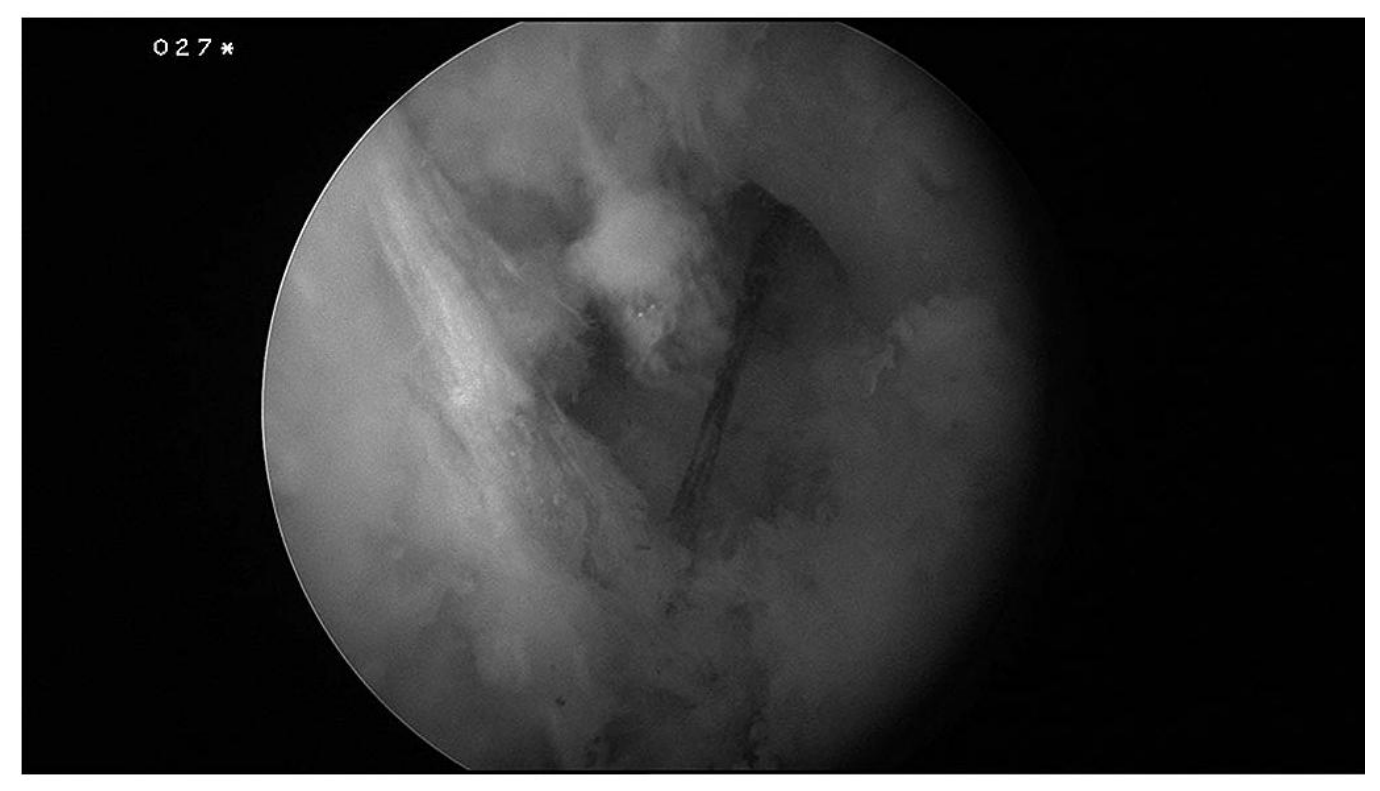

Figure. Intraoperative image demonstrating the orientation and position of the femoral tunnel in the ACL reconstruction 
Table 1. Summary of the Demographic Characteristics of the 3 Groups

\begin{tabular}{|l|c|c|c|c|}
\hline Characteristics & $\begin{array}{c}\text { BPTBAu, } \\
\text { Mean (SD) }\end{array}$ & $\begin{array}{c}\text { BPTBAll, } \\
\text { Mean (SD) }\end{array}$ & $\begin{array}{c}\text { HTAu, } \\
\text { Mean (SD) }\end{array}$ & P \\
\hline Age, y & $28.8(7.1)$ & $25.4(7.3)$ & $26.9(10.0)$ & 0.65 \\
\hline Height, cm & $175.3(5.7)$ & $173.1(7.2)$ & $174.3(8.4)$ & 0.72 \\
\hline Weight, kg & $77.5(12.4)$ & $72.6(14.9)$ & $75.8(12.4)$ & 0.66 \\
\hline BMI, kg/m ${ }^{2}$ & $25.1(2.8)$ & $24.1(3.6)$ & $24.8(2.3)$ & 0.61 \\
\hline \multicolumn{4}{|l}{ BMI, body mass index. } \\
\hline
\end{tabular}

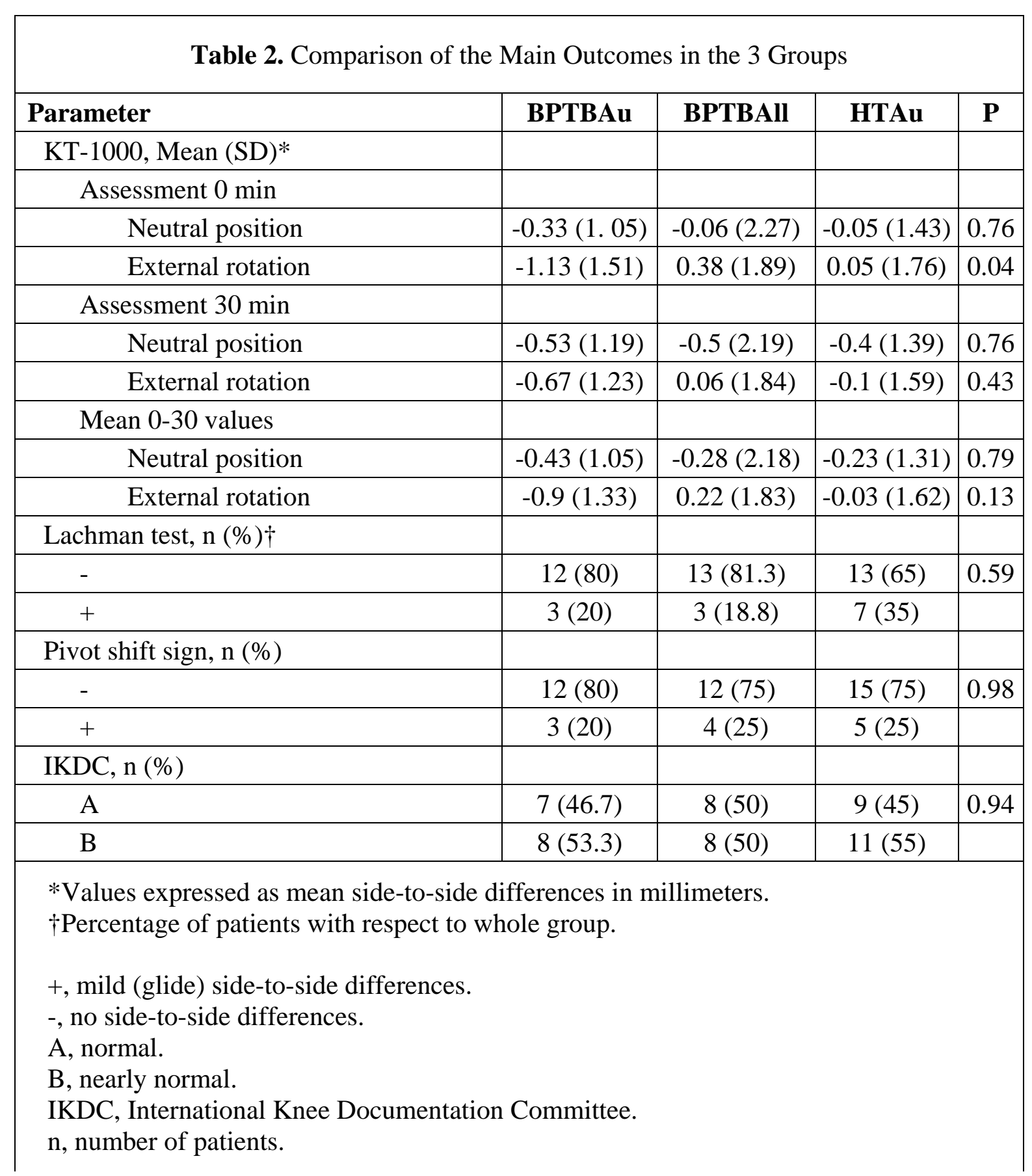


Table 3. Comparison of Patient-Reported Outcomes in the 3 Groups

\begin{tabular}{|c|c|c|c|c|}
\hline Parameter & ВРТВAu & BPTBAll & HTAu & $\mathbf{P}$ \\
\hline \multicolumn{5}{|c|}{ Symptoms, n (\%)* } \\
\hline AKP & $4(26.7)$ & $1(6.3)$ & $2(10)$ & 0.78 \\
\hline Swelling & $0(0)$ & $0(0)$ & $0(0)$ & 0.13 \\
\hline Crepitation & $3(20)$ & $5(31.3)$ & $4(20)$ & - \\
\hline Instability & $1(6.7)$ & $0(0)$ & $1(5)$ & - \\
\hline \multicolumn{5}{|c|}{ VAS satisfaction $\dagger$} \\
\hline Mean (SD) & $8.5(1.3)$ & $9(1.1)$ & $8.9(1.8)$ & 0.28 \\
\hline CI (95\%) & $7.7-9.2$ & $8.5-9.7$ & $8.2-9.9$ & \\
\hline \multicolumn{5}{|c|}{$\begin{array}{l}\text { *Percentage of patients with respect to the whole group. } \\
\text { †Assessed in a } 0 \text { to } 10 \text { scale, where } 0 \text { is worst result. } \\
\text { AKP, anterior knee pain; CI, confidence interval; n, number of patients; VAS, } \\
\text { visual analogue scale. }\end{array}$} \\
\hline
\end{tabular}

Table 4. Comparison of Strength Assessment in the 3 Groups

\begin{tabular}{|c|c|c|c|c|}
\hline Parameter & BPTBAu & BPTBAll & HTAu & P \\
\hline IKS $\dagger$ & & & & \\
\hline Hamstrings, 60\% & & & & \\
\hline Mean (SD) & $6(23.8)$ & $2.2(33.7)$ & $1.8(11)$ & 0.62 \\
\hline CI (95\%) & -8.8 to 20.7 & -14.4 to 18.7 & -3.6 to 7.2 & \\
\hline Hamstrings, 180\% & & & & \\
\hline Mean (SD) & $4.6(28.4)$ & $-3.7(32.3)$ & $-6.4(17.1)$ & 0.37 \\
\hline CI (95\%) & -12.2 to 21.3 & -20.6 to 13.2 & -14.8 to 2 & \\
\hline Quadriceps, 60\% & & & & \\
\hline Mean (SD) & $-7(22.8)$ & $6(29)$ & $1.2(26)$ & 0.32 \\
\hline CI (95\%) & -20.4 to 6.5 & -8.3 to 20.2 & -11.5 to 14 & \\
\hline Quadriceps, 180\% & & & & \\
\hline Mean (SD) & $-3(20.4)$ & $-12.6(42.1)$ & $-5.3(17.2)$ & 0.44 \\
\hline CI (95\%) & -15.6 to 9.6 & -34.6 to 9.44 & -13.8 to 3.1 & \\
\hline
\end{tabular}

†Percentage of deficit of peak torque between the operated and healthy leg for both quadriceps and hamstrings.

CI, confidence interval.

IKS, isokinetic knee strength.

$\mathrm{n}$, number of patients. 